\section{Reichen Sie Ihre Abstracts ein!}

\author{
Der Allergiekongress 2007 in Lübeck bietet eine ausgezeichnete \\ Plattform für den wissenschaftlichen Austausch. Sie können mit Ihrer \\ Präsentation wichtige Akzente setzen und ihre aktuellen Ergebnisse \\ einer Zuhörerschaft zur Diskussion stellen, die die verschiedensten \\ Fachrichtungen umfasst.
}

Z um zweiten Mal findet ein Gemeinsamer Deutscher Allergiekongress der drei Gesellschaften Ärzteverband Deutscher Allergologen (ÄDA), Deutsche Gesellschaft für Allergologie und klinische Immunologie (DGAKI) und Gesellschaft für Pädiatrische Allergologie und Umweltmedizin (GPA) statt, diesmal vom 26. bis zum 29. September 2007 in Lübeck. Federführend ist in diesem Jahr turnusgemäß die DGAKI. Auch für diesen Kongress ist ein umfangreiches wissenschaftliches Programm geplant. Aktuelle Informationen sind auf der Kongress-Homepage www.allergie-kongress.de zu finden.

Das Gerüst des Kongresses bilden

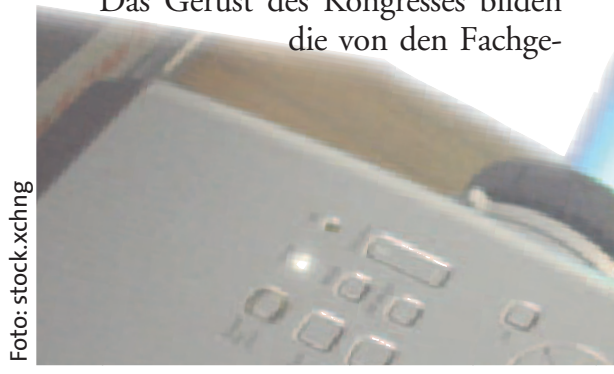

sellschaften organisierten Plenarsitzungen, Symposien und Seminare. Stark sind wir an Ihren Beiträgen interessiert, die Sie über Abstracts zu den Topics Asthma, Urtikaria, Rhinitis, Entzündungszellen und Mediatoren, Nahrungsmittelallergie, Arzneimittelallergie, Insektengiftallergie, Inhalationsallergie und Allergene, Berufsallergien, Allergiediagnostik, Immuntherapie und Kasuistiken u. a. einreichen können.

Die Abstracteinreichung erfolgt elektronisch über das Internet, eine Schritt-für-Schritt-Anleitung dazu finden Sie auf der rechten Seite. Annahmedie eingereichten Abstracts sichten und in die gleichwertigen Kategorien freie Vorträge und Poster einteilen. Die freien Vorträge sind für Donnerstag und Freitag des Kongresses zwischen 17.15 und 18.15 Uhr vorgesehen. Es ist eine Redezeit von sieben Minuten mit zwei schluss ist der 31. Mai 2007. Das regionale Organisationskomitee wird dann
Minuten Diskussion geplant. Die Anzahl der Vortragssitzungen richtet sich in einer bestimmten Bandbreite nach der Anzahl der eingereichten Vorschläge. Die Poster sollten bereits am Donnerstag vor 9.00 Uhr aufgehängt werden. Die Posterbesichtigungen finden am Sonnabend $\mathrm{ab}$ 11.30 Uhr statt. Die Anwesenheit der vortragenden Autoren ist in der Zeit von 12.20-13.15 Uhr vorgesehen. Die Autoren sollten darauf eingerichtet sein, die wichtigsten Inhalte ihres Posters der Begehungskommission in drei Minuten erklären zu können.

Es ist eine Auszeichnung der besten Kurzvorträge und Posterpräsentationen mit jeweils $1.000 €$ geplant. Die Verleihung der Preise und Urkunden ist am Sonnabend in der Plenarsitzung 4 in der Zeit zwischen 15.15 und 17.15 Uhr geplant. Zusätzlich werden alle angenommenen Abstracts in einer Sonderausgabe des Allergo Journal pünktlich zum Kongress publiziert.

Mit dieser Planung hoffen wir, den Allergiekongress 2007 gerade auch für die Nachwuchsforscher attraktiv zu gestalten, da allen ausgewählten Abstracteinsendern die Möglichkeit gegeben wird, ihre Arbeit auch mündlich präsentieren zu können. Wir würden uns freuen, Sie im September in Lübeck begrüßen zu dürfen.

Dr. Wolf-Meinhard Becker, Tagungspräsident, Borstel Priv-Doz. Dr. Regina Fölster-Holst, Kiel, für das regionale Organisationskomitee

Zweiter Gemeinsamer Deutscher Allergiekongress 2007 auf einen Blick

Kongresstermin

26.-29. September 2007

\section{Kongressort}

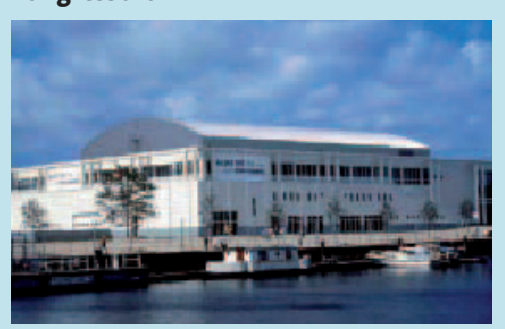

Lübecker Musik- und Kongresshallen, Willy-Brandt-Allee 10, 23554 Lübeck

Wissenschaftliche Leitung

Dr. rer. nat. Wolf-Meinhard Becker, Borstel

\section{Veranstalter}

Ärzteverband Deutscher Allergologen (ÄDA)

Deutsche Gesellschaft für Allergologie und klinische Immunologie (DGAKI)

Gesellschaft für Pädiatrische Allergologie und Umweltmedizin (GPA)

vertreten durch die AKM Allergiekongress und Marketing $\mathrm{GmbH}$, Vohburger Straße 13, 80687 München

Tel.: (o 89) 54662968

Fax: (o 89) 583824

E-Mail: AKMGmbH@t-online.de
Kongressorganisation und Auskunft Service Systems GbR, Blumenstraße 14, 63303 Dreieich

Tel.: (о 61 03) 63657

Fax: (о 61 03) 67674

E-Mail:mail@sersys.de

Kongress-Website

www.allergie-kongress.de

Vorprogramm

Das Vorprogramm kann bei der Kongressorganisation angefordert werden. Eine tabellarische Übersicht findet sich auch auf Seite 174.

\section{Abstract-Einreichung}

Wissenschaftliche Beiträge können von 1. April bis 31. Mai 2007 online eingereicht werden - Anleitung siehe rechte Seite. 